\title{
A02 Fluid Sampling and Analysis
}

John Williams, The Petroleum Institute

Why are fluid properties so important?

- Exploration is required to give an estimate for reserves, and economic valuations requires knowledge of fluid type and quality

- WTI assumes a slightly compressible fluid, and special methods are required for gases, and possibly very volatile oils.

- WTI determines $\mathrm{kh} / \mathrm{mu}$ so a reliable value of viscosity is needed to obtain permeability.

How do we identify the type of fluid?

- Before we drill a well - possibly from seismic, or data from an offset well, or else from correlations

- During drilling - from mud logs (interpretations vary)

- From wireline formation testers - pressure gradients, simple analyses, some properties downhole; problems of contamination; sample analysis

- From well tests - surface measurements such as GOR, oil gravity and colour (but rules are arbitrary, and vary)

- Laboratory studies of samples - most accurate, but takes weeks or months.

How are important properties measured in the laboratory?

- Saturation pressure $(\mathrm{Pb}$ or $\mathrm{Pd})$

- Oil formation volume factor

- Viscosity

- Increasing use of EOS modelling

Which are the big challenges?

- Getting representative samples of the reservoir fluids

- Many reservoirs have compositional gradients

- Production damage caused by reservoir fluids such as scales or asphaltene deposits

- Measuring concentrations of reactive or trace components

What are the risks if fluid properties are wrong?

- Reserve estimates and economic models will be erroneous

- Extra wells or increased pipeline capacity may be required

- Plant design may not be compatible

- Sales contracts may nor be met

- Government rules may not allow production 